\title{
Expert
}

\section{Fool me once, shame on you; fool me twice, shame on me! A perspective on the emerging world of percutaneous heart valve therapy}

Michael Mack, MD

From the Baylor Healthcare System, Cardiopulmonary Research Science Technology Institute, Dallas, Tex.

Presented at the Florida Valve Symposium 2008, March 7-9, 2008, St Petersburg, Fla.

Received for publication May 24, 2008; accepted for publication June 2, 2008.

Address for reprints: Michael Mack, MD, Baylor Healthcare System, Cardiopulmonary Research Science Technology Institute, Dallas, TX 75230 (E-mail: mmack@csant. com).

J Thorac Cardiovasc Surg 2008;136:816-9 $0022-5223 / \$ 34.00$

Copyright $\odot 2008$ by The American Association for Thoracic Surgery

doi:10.1016/j.jtcvs.2008.06.010
$\mathrm{I}$ $\mathrm{n}$ the late 1970s, catheter-based therapy for the percutaneous management of patients with coronary artery disease (CAD) was introduced. At that time, coronary artery bypass surgery (CABG) was the sole interventional treatment available for patients with CAD and offered superior outcomes to medical therapy in many patients. ${ }^{1}$ This new interventional but less invasive treatment, percutaneous coronary intervention (PCI), was met with skepticism, disdain, and dismissive arrogance from within the surgical community. Attitudes manifested by statements such as "we have a superior procedure," "angioplasty will never work," or "outcomes are being compromised" only served to foster complacency and stifle surgical innovation. This comfort in complacency within the surgical community was further reinforced by the annual increase in CABG procedural volume that continued for 20 years after the introduction of catheter-based therapy ${ }^{2}$ (Figure 1). However, the field of PCI progressed rapidly, catalyzed by incremental improvements in technique and technology including steerable catheters, stents, drug-eluting stents, and adjuvant pharmacology. These advancements both decreased procedural morbidity and improved outcomes so that within 10 years after the first interventional coronary procedure was reported, procedural volume of PCI eclipsed that of CABG. So much has the treatment paradigm continued to shift that currently in clinical practice, both interventionalists and their patients often view CABG as the procedure of last resort; the default treatment decision is frequently "if it can't technically be stented, then we'll have to do surgery."

We are now just over 5 years into the brave new world of transcatheter therapy of valvular heart disease, and there are certain similarities to the changing practice paradigm that we witnessed in the early years of PCI in the management of patients with CAD. Since the first report of successful percutaneous aortic valve implantation, all four cardiac valves have now been successfully treated by catheter techniques. ${ }^{3}$ There is now regulatory approval for the commercial sale of two devices for the treatment of aortic stenosis, and one device for the management of mitral insufficiency is in a US pivotal trial. ${ }^{4,5}$ Is this the history of coronary intervention being repeated, that is, déjà vu all over again? There is enough evidence already that this is indeed the case and that, as surgeons, we should learn the lessons of the past.

It is instructive to first view a snapshot of the first 5 years of this new therapy, examine the accomplishments, assess the current barriers, and then suggest how to better create and participate in the future. The pioneering work of Lurz, Bonhoeffer and associates ${ }^{6}$ has resulted in excellent results in the transcatheter management of children and young adults with degenerated right ventricular outflow tract conduits. A total of 417 implants have now been performed with a bovine jugular valve sutured in a nitinol self-expanding stent, the Melody Transcatheter Valve (Medtronic, Inc, Minneapolis, Minn. Results are available on the first 301 patients with 294 (97.7\%) 


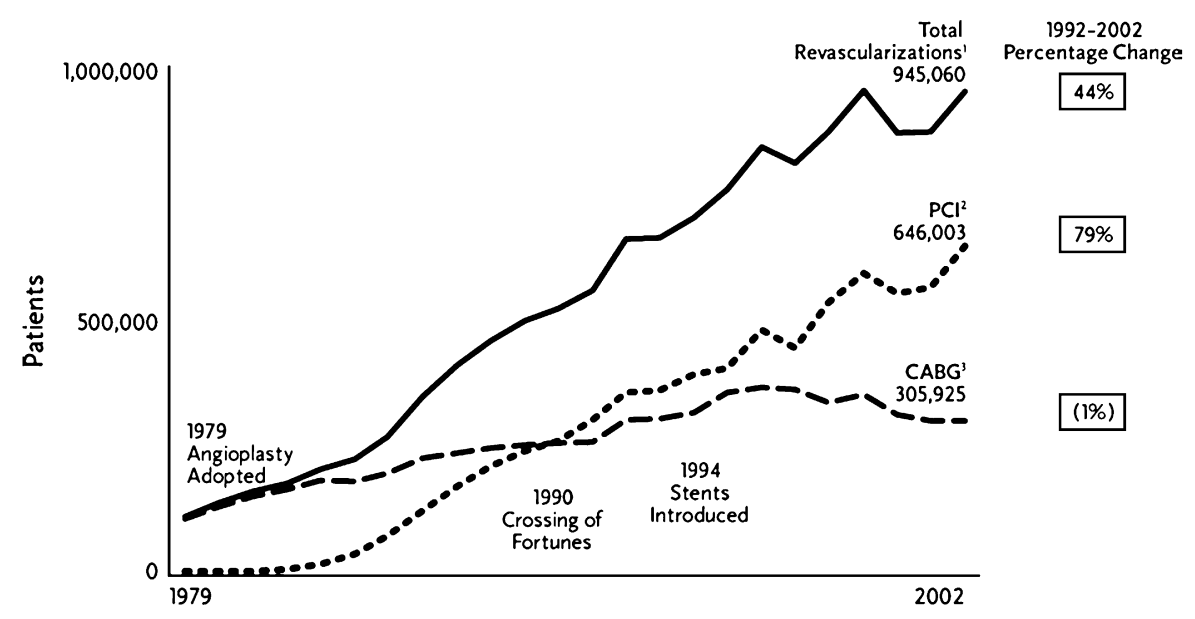

Figure 1. Procedural volumes of coronary revascularization. $P C l$, Percutaneous coronary intervention; CABG, coronary artery bypass grafting. (Reproduced with permission from The Advisory Board Company, Future of Cardiac Surgery-2004, (C) The Advisory Board Company, 2004.)

successful implantations, 284 (94.4\%) treated patients alive, and only $16(5 \%)$ implanted valves explanted. This valve has received CE Mark approval for commercial sale in Europe and is currently in an expanded US feasibility trial. Most procedures have been performed by an antegrade transfemoral venous approach, although a few procedures have been performed through a right ventricular transapical access. Challenges include treatment of patients in need of larger size valves and those with native pulmonary valve disease and transannular patches.

The first report of a transcatheter aortic valve implantation was by Cribier and colleagues ${ }^{3}$ in 2002 . The initial procedures were performed by an antegrade, femoral, transseptal venous approach; however, the ability to reproduce similar results in other centers was an issue. Two other approaches were developed to address this-a retrograde transfemoral, arterial approach and an antegrade transapical approach. The transfemoral approach was facilitated by the development of a flexible delivery catheter capable of negotiating the aortic arch and traversing the stenotic valve in a retrograde fashion. The early developmental work of this approach was by Webb and coworkers ${ }^{4}$ from Vancouver, who now have the largest experience. The transapical procedure was largely developed by Walther and colleagues ${ }^{7}$ in Leipzig, who now have the largest experience with this approach. This device, the Sapien THV (Edwards Lifesciences, Irvine, Calif), is a balloon-deployed tissue valve mounted in a stainless steel stent. This valve has recently been approved for commercial sale in Europe with now more than 1000 valves implanted, currently at a rate of more than 20 per week. Candidates for implantation include inoperable or high-risk operable patients with procedure mortality ranging between $5 \%$ and $10 \%$ in most centers and 1-year survival between $70 \%$ and $75 \%{ }^{8}$ The US pivotal randomized trial of this valve is underway with enrollment in the 1000-patient trial over $25 \%$ completed and commercial approval expected possibly in 2011 .
A second transcatheter aortic valve, the CoreValve Revalving System (CoreValve, Inc, Irvine, Calif) is a nitinol, self-expanding bovine tissue valve delivered percutaneously with an $18 \mathrm{~F}$ system by transfemoral approach without general anesthesia. ${ }^{5}$ This device has also received regulatory approval for commercial sale in Europe with a like number of implants performed with results generally equivalent to those of the Sapien valve. There is early clinical feasibility demonstrated with at least three other catheter-delivered aortic valves. The Direct Flow Medical Valve (Direct Flow Medical, Santa Rosa, Calif) has been successfully implanted in 11 patients in Germany, the Sadra Lotus Valve (Sadra Medical, Campbell, Calif) in 3 patients, and the AorTx (Hansen Medical, Mountain View, Calif) in 3 patients. There is also a pipeline full of innovative valve designs that promise to overcome some of the limitations of the early-generation devices, including enhanced deliverability, retrievability, and the ability to be repositioned. As enhanced delivery, safety, and valve durability are demonstrated, indication for implantation into lower-risk patients is inevitable.

Although promising in the early stages of clinical application, the path to the demonstration of clinical efficacy has been a more arduous one for the percutaneous treatment of mitral valve disease. Demonstration of proof of principle for the treatment of mitral regurgitation (MR) from both intrinsic and functional etiologies has occurred. Devices that replicate the Alfieri "edge-to-edge" approach have been used primarily for degenerative disease, although there is some early evidence that functional MR could also possibly be treated by this technique. ${ }^{9}$ One device, the Evalve MitraClip (Evalve, Inc, Menlo Park, Calif), has completed a feasibility trial, a high-risk registry, and is nearing completion of enrollment in a pivotal randomized trial comparing the percutaneous clip to conventional mitral valve repair. A second device, the Edwards Mobius, which performed an edge-to-edge approximation using a suture-based system rather than a clip, 
has been shelved after disappointing results in a small feasibility trial.

A host of devices to remodel the mitral annulus either via the coronary sinus or directly in patients with functional MR are in clinical investigation. Although isolated proof of concept, safety, and some early clinical efficacy has been demonstrated with a number of catheter-delivered devices, progress has been slower than anticipated. One issue is lack of certainty regarding the role for correction of MR in heart failure even under optimal open surgical conditions. This uncertainty stems from the fact that MR in heart failure is due to failure of leaflet coaptation from ventricular dilation and apical lateral distraction of the papillary muscles tethering the mitral leaflets and only secondarily to annular dilation. Further complicating factors include the variability in the relationship between the coronary sinus and the posterior mitral annulus and the intervening coronary artery that frequently occurs between the coronary sinus and the mitral annulus. Other devices that have preclinical proof of principle and/or early clinical experience include those that do not use the coronary sinus but remodel the annulus directly either by suture, device, or by radiofrequency shrinkage. Still other devices are designed to remodel the annulus secondarily by first reconfiguring the shape of the left ventricle (iCoapsys; Myocor, Maple Grove, Minn) or left atrium (PS3; Ample Medical, Foster City, Calif). Other devices are focused on correcting MR by placement of artificial cords to the mitral leaflets by a minimally invasive approach (Neochord Inc, Wayzata, Minn; Transcardiac Therapeutics, Atlanta, Ga). An even more intriguing concept includes that of percutaneous mitral valve replacement (EndoValve Inc, Princeton, NJ).

The progress in the introduction of catheter-based therapy has been more rapid than expected for aortic stenosis, slower than anticipated for MR, and has achieved excellent early results in right ventricular conduits. It must be remembered that these results have been achieved with early-stage devices and delivery systems in clinical situations in which both appropriate patient selection and procedural techniques are evolving. There is a pipeline full of the next generation of devices backed by strong financial investment and creative engineering minds that will likely solve the challenges encountered by these first-generation devices.

How do we as a surgical community react and adapt to this potential change in the management of valvular heart disease that is bound to affect our current practice? First, we should not become complacent by the fact that currently the number of valve operations is increasing. Just as in the world of coronary revascularization, in which the first two decades witnessed a continuous increase in the annual volume of CABG procedures, we are likely to see the same increase in surgical valve procedures for the foreseeable future. There are many reasons for this, including the wider availability of diagnostic imaging, greater recognition that valvular heart disease is underdiagnosed and undertreated, and new studies supporting treatment of asymptomatic patients. The potential of percutaneous therapy has also brought added focus to this field. However, just as the annual number of coronary procedures performed by a percutaneous approach exceeded those done by a surgical one for a decade after introduction, the same relative growth rates are likely to be seen in the treatment of valve disease as it is affected by the emerging field of transcatheter valve therapy. Whether this shift will occur within a decade or longer is impossible to predict owing to the unpredictability of many variables including device iteration, clinical trial results, and regulatory and reimbursement issues; however. this shift will indeed happen!

We as a cardiac surgical community have a choice; we can stand by on the sidelines and witness this shift with skepticism and disparagement as we did PCI, or we can choose to participate. The role that surgeons and the surgical community are going to play is to a large degree up to us. If we want to act like a "guild" with a protectionist attitude toward our members, then we are likely to see the size of our specialty decrease and our role in the management of valvular heart disease diminish. However, if we wish to maintain a prominent role in the management of valvular heart disease, then we need only look as far as our sister specialty of vascular surgery to learn how to adapt. Vascular surgeons evaluated the changing treatment paradigms of vascular disease management as catheter therapy became more versatile and advanced. They elected to learn the skill sets necessary to participate in catheter-based therapy. Whereas 10 years ago vascular surgery was a specialty performing predominantly open vascular operations, most successful vascular surgery practices are now largely endovascular ones. The specialty as a whole and surgeons as individuals took it upon themselves to assess the treatment landscape, learn new skill sets heretofore lacking, embrace change, and critically analyze results. As a result, vascular surgeons now possess the ability to choose an open or endovascular procedure or a hybrid of both depending on specific patient needs. I suggest that it would be prudent for us as cardiac surgeons to take this approach, work collaboratively with our interventional colleagues who have skill sets and perspectives different from ours, and participate in a constructive manner of developing and implementing new and appropriate treatment paradigms for patients with valvular heart disease.

We have a proud heritage in cardiac surgery that has seen miraculous therapies introduced that have benefited our patients over the past half century. However, the specialty of cardiac surgery is currently in a crisis mode; training positions are going unfilled, the workforce is disheartened, and the best and the brightest are no longer attracted to our field. It is time to reinvent our specialty and ourselves. The leadership of four major organizations in cardiothoracic surgery, The American Association for Thoracic Surgery (AATS), the American Board of Thoracic Surgery (ABTS), The Society of Thoracic 
Surgeons (STS) and the Thoracic Surgery Foundation for Research and Education (TSFRE) have recently set forth a mandate for change in which graduate and postgraduate education will be totally revamped to address the current situation. As critical as the opportunities that this initiative will create, we as individuals cannot wait for this to be fixed for us. Change must come from within. We must critically analyze our own situations, keep what is good, discard that which is arcane and outmoded, have the wisdom to know the difference, and embrace the opportunities presented.

Nowhere else will that mandate for change and the adoption of new treatment paradigms and skill sets be more evident that in the field of valvular heart disease. We have already seen this shift, not only in the interventional management of coronary artery disease but also in vascular disease, general surgery, and orthopedic surgery. Patients will choose the less invasive approach over a more invasive one even when they understand that the efficacy and duration of the treatment benefit may not initially be as great with the less invasive approach. However, progress is inexorable and eventually the benefits of new, less invasive procedures equal or surpass those of their open predecessors, for example, laparoscopic cholecystectomy and arthroscopy.

We must not be complacent regarding our role as surgeons in valvular heart disease. We have superlative outcomes manifest by decreasing mortality in the face of an aging population with increasingly complex disease, increasing valve repair rates, and higher referral rates, especially for the management of asymptomatic disease. However, just as in the dawn of the age of PCI, we have the choice; we can rest on our laurels, stand by our outcomes, and disparage the new treatments being invented. Alternatively, we can learn from the past, embrace change, gain the skill sets needed to participate and thrive, and be part of the process rather than be unengaged bystanders.

Fool me once, shame on you; fool me twice, shame on me!

\section{References}

1. Coronary Artery Surgery Study (CASS): a randomized trial of coronary artery bypass surgery. Survival data. Circulation. 1983;68:939-50.

2. Stuge O, Liddicoat J. Emerging opportunities for cardiac surgeons within structural heart disease. J Thorac Cardiovasc Surg. 2006;132: 1258-61.

3. Cribier A, Eltchaninoff H, Bash A, Borenstein N, Tron C, Bauer F, et al. Percutaneous transcathether implantation of an aortic valve prosthesis for calcific aortic stenosis. Circulation. 2002;106:3006-8.

4. Webb JG, Chandavimol M, Thompson CR, Ricci DR, Carere RG, Munt BI, Buller CE, Pasupati S, Lichtenstein S. Percutaneous aortic valve implantation retrograde from the femoral artery. Circulation. 2006;113:842-50.

5. Grube E, Schular G, Buellesfeld L, Gerckens U, Linke A, Wenaweser B, et al. Percutaneous aortic valve replacement for severe aortic stenosis in high-risk patients using the second- and current third-generation self-expanding CoreValve prosthesis: device success and 30-day clinical outcome. J Am Coll Cardiol. 2007;50:69-76.

6. Lurz P, Coats L, Khambadkone S, Nordmeyer J, Boudjemline Y, Schievano S, et al. Percutaneous pulmonary valve implantation. Impact of evolving technology and learning curve on clinical outcome. Circulation. 2008;117:1964-72.

7. Walther T, Simon P, Dewey T, Wimmer-Greinecker G, Volkmar F, Kasimir M, et al. Transapical minimally invasive aortic valve implantation. Circulation. 2007;116(11 Suppl):I240-5.

8. Webb JG. Percutaneous aortic valve replacement will become a common treatment for aortic valve disease. J Am Coll Cardio Intervent. 2008;1: 122-6.

9. Feldman T, Leon MB. Prospects for percutaneous valve therapies. Circulation. 2007;116:2866-77. 